Präv Gesundheitsf 2019 · 14:407-413 https://doi.org/10.1007/s11553-019-00705-8 Eingegangen: 9. Dezember 2018

Angenommen: 29. Januar 2019

Online publiziert: 14. Februar 2019

(c) Der/die Autor(en) 2019

\author{
Jan Kramer ${ }^{1,2,3} \mathbb{D} \cdot$ Uli Früh $^{4}$ \\ ${ }^{1}$ Arbeitsgemeinschaft Versorgungsforschung, Verband Akkreditierte Labore in der Medizin (ALM) e.V., \\ Berlin, Deutschland \\ ${ }^{2}$ LADR Der Laborverbund Dr. Kramer \& Kollegen, Geesthacht, Deutschland \\ ${ }^{3}$ Medizinische Klinik I, Universität zu Lübeck, Lübeck, Deutschland \\ ${ }^{4}$ WCG Consulting, Reutlingen, Deutschland

\section{Prävention von Darmkrebserkrankungen}

\section{Bericht der Akkreditierten Labore in der Medizin über den Einsatz immunologischer fäkaler okkulter Bluttests}

\section{Hintergrund}

Kolorektale Karzinome (KRK) sind weltweit die dritthäufigste Krebserkrankung [5]. In Deutschland erkrankten im Jahr 2013 neu 28.360 Frauen und 34.050 Männer bei insgesamt 25.693 durch ein KRK begründeten Todesfällen [32]. Die Inzidenz steigt ab dem 50. Lebensjahr an. Durch ein präventives Screening ab diesem Alter sollen Adenome als Vorstufen oder KRK frühzeitig entdeckt werden. Die Inzidenz von Dickdarmkrebs sinkt in Deutschland seit 2004, während fortgeschrittene Vorstufen häufiger durch die im Jahr 2002 eingeführten Früherkennungskoloskopien detektiert werden. Wird die Koloskopie abgelehnt, kann der sog. fäkale okkulte Bluttest (FOBT) im Stuhl eingesetzt werden.

\section{Anerkennung und Nutzung von Früherkennungsmaßnahmen bei Dickdarmkrebs}

In der Bevölkerung kennen $>80 \%$ das Angebot zur Krebsfrüherkennung [32]. Allerdings nutzen es nur $67 \%$ der Frauen und $40 \%$ der Männer. Gesundheitspolitisches Ziel ist, diese Untersuchungen zu steigern. Dies soll über bessere Informationsangebote erreicht werden.

In der Studie zur Gesundheit Erwachsener in Deutschland (DEGS1) gaben für die Jahre 2008-2011 51,9\% der An- spruchsberechtigten (55,5\% der Männer und $48,0 \%$ der Frauen) an, einen FOBT im Stuhl in den letzten 1-2 Jahren genutzt zu haben [26]. Nach den Berechnungen des Zentralinstituts (ZI) für die kassenärztliche Versorgung aus dem Jahr 2016 lag die Inanspruchnahme des FOBT in den Jahren 2010-2011 bei 31,3\% bzw. 23,2\% der jeweils anspruchsberechtigten Frauen bzw. Männern [31]. Die Schätzungen für 2013-2014 liegen bei 27,0\% für Frauen und 18,8\% für Männer und damit niedriger als in den Vorjahren, was mit einer Zunahme der durchgeführten Koloskopien zusammenhängen kann.

Laut der DEGS1-Studie wurde bei $57,7 \%$ der $\geq 55$-Jährigen (Frauen 58,6\%, Männer 56,7\%) in den letzten 10 Jahren eine Koloskopie durchgeführt [26]. Das ZI weist eine Steigerung der Koloskopien über einen mehrjährigen Beobachtungszeitraum aus [31]. Die Koloskopie wurde von $17,4 \%$ bzw. $17,9 \%$ in den Jahren von 2003-2011 der anspruchsberechtigten Frauen bzw. Männer genutzt. Die aktuellen Schätzungen liegen für den Zeitraum 2003-2014 mit 20,4\% bei den Frauen und 20,8\% bei den Männern höher. Die Daten zeigen eine Zunahme der Koloskopie mit dem Alter, allerdings nur bis zur Altersgruppe von 70-74 Jahren.

Wurden Studienteilnehmer in Deutschland nach Gründen für eine Nichtteilnahme an der Früherkennungskoloskopie gefragt [27], zeigte sich ein Bedarf an besseren Informationen. Beispiele für verständliche Informationen zum Thema Darmkrebsfrüherkennung finden sich auf www.gesundheitsinformation.de, krebsinformationsdienst.de oder vorsorgeonline.de.

Sinnvoller Einsatz fäkaler okkulter Bluttests im Stuhl

KRK bluten in das Darmlumen. Dieses in der Regel nicht sichtbare Blut kann mit FOBT im Stuhl nachgewiesen werden. Eine wiederholte Testung führt dabei $\mathrm{zu}$ einer zuverlässigen Erkennung von KRK [1]. Hämoglobin färbt bei Guajakharz-basierten FOBT (gFOBT; z.B. Hämoccult $^{\circledR}$, Beckman Coulter, USA) nach Zugabe von Wasserstoffperoxid das imprägnierte Filterpapier blau. In 3 Stuhlgängen waren bei diesen herkömmlichen gFOBT je 2 Proben pro Stuhl auf 2 Testfelder aufzutragen und auf okkultes Blut zu testen [10]. Für Patienten, die präventiv mindestens einmal einen FOBT durchgeführt hatten, konnte eine Senkung der KRK-bedingten Mortalität um bis zu $30 \%$ nachgewiesen werden [12, 23, 25]. Die Testung einmal pro Jahr ist der 2-jährigen Testung überlegen [19]. Bereits ein positives Testfeld macht eine Koloskopie zur Tumorsuche erforderlich. Allerdings wurden selbst in Studien nur 64 bis $<90 \%[21,28]$ aller 


\section{Übersicht}

\section{Tab. 1 Darmkrebsfrüherkennung von GKV-Patienten}

Früherkennungskoloskopie als Goldstandard

$\geq 50$. Lebensjahr 2 Früherkennungskoloskopien im Abstand von 10 Jahren

$\geq 65$. Lebensjahr Jede $\geq 65$. Lebensjahr durchgeführte gilt als 2 . Koloskopie

Prävention mit iFOBT, falls keine Koloskopie genutzt wird

$\geq 50$. Lebensjahr Jährlich (1x/Jahr)

$\geq 55$. Lebensjahr Alle 2 Jahre $(1 \mathrm{x} / 2$ Jahre)

Merke:

Seit dem 1. April 2017 ersetzt der iFOBT den gFOBT

Die Ausführungsrichtlinien des Gemeinsamen Bundesausschusses fordern Qualitätsstandards des iFOBT im Stuhl sowie die automatisierte Durchführung in zentralen medizinischen Laboratorien mit Akkreditierung

Die Durchführung des iFOBT in der Arztpraxis wird durch den Gemeinsamen Bundesausschuss strikt abgelehnt

iFOBT Immunologischer fäkaler okkulter Bluttest, gFOBT Guajakharz-basierter immunologischer fäkaler okkulter Bluttest, GKV gesetzlich krankenversichert

${ }^{a}$ CAVE: Bei familiärem Risiko ist bereits ab dem 45. Lebensjahr die Koloskopie angeraten

Patienten mit einem positiven FOBT koloskopiert. Nachteilig am gFOBT ist eine nur mäßige Sensitivität für Karzinome und eine geringe Sensitivität für Adenome [7]. Eine falsche Durchführung beeinflusst die Spezifität des gFOBT: der Test kann falsch-positive Ergebnisse liefern, da er z. B. auch auf tierisches Blut aus rohen Fleischwaren reagiert.

\section{Vorteile quantitativer}

immunologischer fäkaler okkulter Bluttests im Stuhl

Immunologische FOBT (iFOBT) basieren hingegen auf spezifischen Antikörpern gegen humanes Hämoglobin. Im Gegensatz zu den gFOBT können iFOBT automatisiert durchgeführt werden. Auch im Ausland werden iFOBT in Screeningprogrammen eingesetzt [24]. Verfügbare Testsysteme (jeweiliger Anteil an den hier vorgestellten Daten in Klammern) sind z.B. zur Vollautomatenanalyse OC Sensor ${ }^{\circledR}$ (Eiken Chemical, Japan/Mast Diagnostica, Deutschland; 47\%) als FIT-Screening, FOB Gold Sentinel ${ }^{\circledR}$ (Sysmex, Japan; $24 \%)$ als Sentifit ${ }^{\circledR}$ sowie NS-Plus C15 Otsuka $^{\circledR}$ (Care diagnostica, Deutschland; $0 \%$ ) als CAREprime ${ }^{\circledR}$. ELISA-Tests, z.B. auf dem ELISA-Automaten DSX ${ }^{\circledR}$ (Dynex Technologies, USA) analysiert, sind RidaScreen ${ }^{\circledR}$ Haemoglobin (R-Biopharm, Deutschland; $3 \%$ ), ScheBo ${ }^{\circledR} \mathrm{Hb}$ Smart ELISA (Biotech, Deutschland; $0 \%$ ) und Hämoglobin ELISA (Immundiagnostik, Deutschland; 26\%).
KRK und auch fortgeschrittene Adenome werden durch iFOBT besser erkannt [3, 7, 11, 20, 33]. Beispielsweise konnte eine Überlegenheit des eingesetzten iFOBT-OC-Sensors ${ }^{\circledR}$ gegenüber dem Hämoccult ${ }^{\circledR}$-Test in großen Studien gezeigt werden $[13,22]$. Die Zahl der Intervallkarzinome, die nach einer negativen Früherkennung und vor der nächsten regulären Untersuchung entdeckt werden, ist ein wichtiger Indikator für die Qualität eines Screenings. Umso geringer die Inzidenz von Intervallkarzinomen umso besser ist die Erkennung des KRK. In einer Metaanalyse wurde die Inzidenz von Intervallkarzinomen bei Reihenuntersuchungen verglichen [30]. Auf 100 entdeckte Intervallkarzinome entfielen hierbei 83 auf den gFOBT und 38 auf den iFOBT. Die iFOBT sind damit auch in dieser Hinsicht den gFOBT überlegen.

Die Sensitivität und Spezifität der verfügbaren iFOBT kann allerdings deutlich variieren [14]. Für die Spezifität wird die akzeptable Grenze bei $\geq 90 \%$ angesehen [2]. Bezüglich der Sensitivität besteht die Möglichkeit einer Änderung des Hämoglobinschwellenwertes (Cut-off-Wertes), bei dem ein iFOBT als positiv bewertet wird. Schwellenwerte $<20 \mu \mathrm{g} / \mathrm{g}$ (Rate positiver iFOBT: 5-14\%) zeigten die besten Werte für die gepoolte Sensitivität mit 89 (80-95)\% und gepoolte Spezifität mit 91 (89-93)\% [7, 18]. Der optimale Cut-offWert bleibt aber in Diskussion.

In einer richtungweisenden Studie wurden 9 iFOBT verglichen [9] und alle Tests entdeckten die große Mehrheit al- ler KRK und auch viele Vorstufen. Folgt man den Angaben der Hersteller, ab welchem Wert ein Test als positiv zu werten ist, unterscheiden sich die Häufigkeiten positiver Resultate jedoch deutlich. Erst als die Cut-off-Werte testindividuell angepasst wurden, lieferten alle Tests ähnliche Ergebnisse. Die Steuerungsfähigkeit der iFOBT über Einstellungen dieses Schwellenwertes ist ein weiterer Vorteil, der zukünftig darüber hinaus auch risikoadaptierte Betrachtungen ermöglichen kann.

Aufgrund der besseren Möglichkeiten der Steuerung und Qualitätssicherung (QS) wird ab dem April 2017 ausschließlich der quantitative iFOBT für das Darmkrebsscreening von GKV(gesetzlich krankenversicherte)Patienten eingesetzt ([6], - Tab. 1). Die Europäische Leitlinie (EU LL) zur QS von Krebsfrüherkennungsmaßnahmen empfiehlt für ein Darmkrebsscreening automatisierte und qualitätsgesicherte Verfahren, um analytische und prozessbezogene Unterschiede bei der Anwendung der Tests zu minimieren [4]. Vorgaben zur geforderten Qualität des Einsatzes von iFOBT von GKV-Patienten sind in $\$ 39$ der Krebsfrüherkennungsrichtlinie (KFE-RL) festgehalten [17].

\section{Beachtung der Präanalytik zur Qualitätssicherung des iFOBT}

Sensitivität und Spezifität des iFOBThängen neben der Art der Testdurchführung entscheidend von der Patienteninstruktion zur Probenentnahme ab. Die Ausgabe des Stuhlentnahmesystems soll daher über Ärzte erfolgen [6]. Dies ist für den iFOBT nur zulässig durch Hausärzte, Internisten, Chirurgen, Gynäkologen und Urologen. Bis zum 01.04.2017 wurden in etwa $70 \%$ der gFOBT von Gynäkologen ausgegeben [7]. Für eine höhere Teilnahmerate insbesondere bei Männern sollte der iFOBT möglichst auch von Allgemeinärzten und hausärztlich tätigen Internisten präventiv angeboten werden.

Die Patienten müssen bei der Ausgabe der Tests darauf hingewiesen werden, die Probe am Tag nach der Abnahme wieder in der Praxis abzugeben [6]. Wichtig ist bereits eine Überprüfung der richtigen Abnahme, z. B. kann ein über- 
fülltes Entnahmesystem nicht verwendet werden. Der Arzt hat bei richtig entnommenen Proben zu veranlassen, dass spätestens am nächsten Werktag nach Rückgabe die Weiterleitung an ein Labor erfolgt [6]. Das Entnahmesystem muss eine verständliche Anleitung beinhalten und eine hygienische Stuhlaufnahme sowie die Überführung einer definierten Stuhlmenge in ein Puffersystem sicherstellen, sodass die Probenstabilisierung bei Raumtemperatur mindestens 5 Tage gewährleistet ist [6]. Dennoch besteht ein Restrisiko falsch-negativer Befunde durch Hämoglobinabbau v. a. bei Versand in der warmen Jahreszeit.

Die Stuhlprobe soll unmittelbar nach Eingang im Labor analysiert werden [6]. Die Untersuchung hat mittels automatisierter Verfahren zu erfolgen. Das Labor teilt dem einsendenden Arzt umgehend das Ergebnis mit. Bei einem positiven iFOBT informiert der Arzt in der Praxis den Patienten darüber, dass eine Koloskopie zur Abklärung angeraten ist. Negative Ergebnisse werden gemäß KFERL nur auf ausdrücklichen Wunsch des Versicherten mitgeteilt.

\section{Verbindliche Vorgaben zur Qualitätssicherung bei der Laboranalytik des iFOBT}

Als Voraussetzung sollen iFOBT durch akkreditierte medizinische Labore (DIN EN ISO 15189) erbracht werden, die mindestens 10.000 Stuhlproben pro Quartal oder 40.000 Stuhlproben über das gesamte Jahr mit iFOBT untersuchen [7]. Die EU LL empfiehlt als sog. frühe Performanceindikatoren die Auswertung des Anteils nicht verwertbarer Tests sowie des Anteils positiver Proben an der Probengesamtzahl je nach Testprodukt und Labor [4]. Der Anteil an nicht verwertbaren Proben soll bei maximal 3\% liegen, besser $\leq 1 \%$. Der Anteil an positiven Befunden des iFOBT soll bei $\leq 10 \%$ liegen [7]. Die eingesetzten iFOBT sollen bei der Analyse von nur einer Stuhlprobe eine Spezifität von $\geq 90 \%$ und eine Sensitivität $\geq 25 \%$ für fortgeschrittene Adenome oder KRK besitzen [6]. Zu belegen ist dies durch eine vorherige Studie mit Koloskopie als Referenzverfahren.

Präv Gesundheitsf 2019 · 14:407-413 https://doi.org/10.1007/s11553-019-00705-8

(c) Der/die Autor(en) 2019

J. Kramer · U. Früh

Prävention von Darmkrebserkrankungen. Bericht der

Akkreditierten Labore in der Medizin über den Einsatz immunologischer fäkaler okkulter Bluttests

Zusammenfassung

Hintergrund. Der Artikel gibt eine Übersicht über die Einführung der immunologischen fäkalen okkulten Bluttests (iFOBT) für gesetzlich krankenversicherte (GKV-)Patienten im Rahmen der Früherkennung des Darmkrebses in Deutschland zum 1. April 2017 und beinhaltet den Bericht der medizinischen Labore zum Einsatz dieser präventiven Laboranalytik.

Fragestellung. Der Bericht beantwortet Fragen zur Häufigkeit der Anforderung des iFOBT sowie Qualitätsaspekten der Präanalytik und Analytik auf Basis der Auswertung der Daten und Erfahrungen aus dem Routineeinsatz in der Patientenversorgung.

Material und Methode. Die Auswertung basiert auf anonymisierten Daten der Mitglieder der Arbeitsgemeinschaft Versorgungsforschung der Akkreditierten Labore in der Medizin (ALM) e.V. vom 1. April 2017 bis 30. Juni 2018 und beinhaltet 4.423 .795 von den Laboren ausgegebene iFOBT.
Ergebnisse. Die Rücklaufquote der Tests in die Labore betrug nur $62 \%(n=2.725 .849)$. Zudem waren hiervon 4\% $(n=110.514)$ der iFOBT nicht auswertbar. Dies war im Wesentlichen durch eine nicht sachgerechte Handhabung durch die Patienten begründet. Die Durchführung der iFOBT ist gemäß den Qualitätsvorgaben in den akkreditierten medizinischen Laboren für die Routine in der Darmkrebsfrüherkennung ansonsten etabliert.

Schlussfolgerungen. Eine Optimierung der Durchführung der Früherkennungsmaßnahme muss das Ziel weiterer Maßnahmen im Interesse der Patientenversorgung und Prävention sein. Entsprechende Lösungsansätze für die Praxis werden hier vorgeschlagen.

Schlüsselwörter

Darmkrebsfrüherkennung · Darmkrebsprävention - Darmkrebsvorsorge - Stuhltest . Kolonkarzinom

\section{Prevention of colorectal cancer. Report of accredited laboratories in medicine on the use of immunological faecal occult blood testing}

\section{Abstract}

Background. This review summarizes information about the introduction of immunological faecal occult blood testing (iFOBT) in line with the screening for colorectal cancer in the outpatient area with statutory health insurance since 01 April 2017, in Germany. A field report of medical laboratories in patient care for iFOBT is also included.

Objectives. The report responds to questions about the frequency of iFOBT laboratory orders as well as quality issues regarding preanalytic and analytic topics based on data analysis and practical experience from routine use in patient care.

Materials and methods. The analysis is based on anonymous data of the members from the German Federation of Accredited Laboratories in Medicine (ALM) from 01 April 2017 to 30 June 2018 including 4,423,795 iFOBT provided by the laboratories to the outpatient physicians.

Results. The rate of returned tests to the laboratories by physician's practices was only $62 \%(n=2,725,849)$. In addition, $4 \%$ of the tests were not exploitable mainly due to false handling of the stool sampling by patients. Meanwhile, laboratory test criteria meet the quality requirements.

Conclusions. Optimization of the implementation of the whole medical procedure of $\mathrm{iFOBT}$ is needed for better outcome in patient care. Here, we suggest approaches for practical solutions with defined aims in our concluding remarks.

\section{Keywords}

Cancer screening tests - Preventive medicine . Colorectal cancer - Occult blood · Early diagnosis of cancer 


\section{Übersicht}

\section{Tab. 2 Leistung und Abrechnung des iFOBT bei GKV-Patienten}

Präventive Darm- $\quad$ Ärzte, die den iFOBT präventiv bei ihren Patienten $\geq 50$. Lebensjahr veranlas krebsfrüherkennung

sen (• Tab. 1), rechnen die GOP 01737 (57 Punkte, $6 €$ ) ab.

Die Leistung umfasst die Patientenberatung, Ausgabe, Rücknahme und Weiterleitung des Stuhlprobenentnahmesystems.

Wichtig ist der Vermerk „präventiv“ auf dem Anforderungsmuster 10

Kurative Untersuchungsindikation

Keine zusätzliche Vergütung für den veranlassenden Arzt; Ausgabe, Rücknahme und Weiterleitung in das Labor sind mit der Versicherten- oder Grundpauschale abgegolten.

Es erfolgt der Vermerk "kurativ" auf dem Anforderungsmuster 10

Der Laborarzt im akkreditierten medizinischen Labor rechnet für die Untersuchung der Stuhlprobe wie folgt ab: bei einer präventiven Untersuchung die GOP 01738 (75 Punkte, 7,99€) bzw. bei einer kurativen Untersuchungsindikation die GOP 32457 (5,52€ nach Abzug der Laborquote Q)

FOBT Immunologischer fäkaler okkulter Bluttest, GKV gesetzlich krankenversichert

Der iFOBT kann nur von Ärzten durchgeführt werden, die eine Genehmigung gemäß der Vereinbarung $\mathrm{zu}$ den Laboratoriumsuntersuchungen nach $\S 135$ Abs. 2 des SGB V zur Ausführung und Abrechnung von Leistungen nach den Gebührenordnungspositionen (GOP) in den Abschn. 32.3 und 1.7 des Einheitlichen Bewertungsmaßstabes (EBM) besitzen [6]. Das akkreditierte Labor muss entsprechend den Vorgaben in $\$ 4 \mathrm{a}$ der Medizinproduktebetreiberverordnung ein System der QS etabliert haben und sich regelmäßig an geeigneten externen QS-Maßnahmen für den iFOBT beteiligen. Die den Test im Labor durchführenden Vertragsärzte sind verpflichtet, Quartalsberichte zusammenzustellen und mit der Abrechnung der zuständigen Kassenärztlichen Vereinigung (KV) zu übermitteln [6]. Die Quartalsberichte beinhalten Angaben $\mathrm{zu}$ den verwendeten iFOBT (Produktnamen, Reagenzcharge, Schwellenwerte und ggf. Herstellererklärungen) und den externen QS-Maßnahmen (z.B. Ringversuche). Anzugeben sind auch die Gesamtzahl der untersuchten und der positiven sowie der nicht verwertbaren Proben. Die Quartalsberichte sind Voraussetzung für die Abrechnung des iFOBT. - Tab. 2 informiert über Hintergrundwissen bezüglich Leistungsinhalt und Abrechnung des iFOBT nach dem EBM. Die Quartalsberichte werden von den KVen einrichtungspseudonymisiert einer vom Gemeinsamen Bundesausschuss beauftragten zentralen Stelle zur Auswertung zugeführt.

\section{Ergebnis des iFOBT-Einsatzes in der Datenauswertung medizinischer Laboratorien}

Die AG Versorgungsforschung des Verbands der Akkreditieren Labore in der Medizin (ALM) e. V. hat WCG Consulting als Beratungsunternehmen für das Controlling medizinischer Leistungen beauftragt, eine anonymisierte Datenauswertung der zum 01.04.2017 bis 30.06.2018 durch die ALM-Mitgliedslabore erfassten iFOBT durchzuführen. Beteiligte ALM-Mitgliedslabore sind: Amedes, Labor Berlin, Diagnosticum, Institut für Medizinische Diagnostik (IMD)/Synevo, Labopart, LADR Der Laborverbund Dr. Kramer \& Kollegen, Labdiagnostik, Nordlab, MVZ Labor Dr. Riegel, Sonic, Synlab und Labor Dr. Wisplinghoff. Zukünftige Labordatenauswertungen können durch neue ALM-Mitgliedslabore zusätzlich Daten der Limbach Gruppe sowie der Laborarztpraxis Dres. Walther, Weindel und Kollegen MVZ beinhalten.

Im genannten Zeitraum wurden Daten von 4.423.795 ausgegebenen und 2.725.849 zurückerhaltenden iFOBT ausgewertet (• Abb. 1a). Dies waren allein im Q4/2017 554.370 iFOBT (76\% GOP 01738, $n=421.321 ; 24 \%$ GOP 32457, $n=133.049$ ). Die Menge der ALM-Daten entspricht in etwa $64 \%$ der durch die KBV veröffentlichten Testanzahl in Q4/2017 [15] mit 660.530 Leistungen der GOP 01738. Der Anteil der jeweils durch die ALM-Mitgliedslabore eingesetzten Testsysteme wurde weiter oben bei der Vorstellung einiger Hersteller zusammengefasst. Der An- teil positiver Befunde mit $10 \%$ aller der in ALM-Mitgliedslaboren zurückerhaltenden iFOBT liegt innerhalb der Qualitätsvorgaben (• Abb. 1b).

Allerdings sind Optimierungen in der Anforderung desiFOBT und der Prozessabwicklung notwendig:

- Es werden mehr s ausgegeben, als die Labore zurückerhalten: die Rücklaufquote beträgt im untersuchten Gesamtzeitraum in Summe nur 62\%, für das Q4/2017 $75 \%$. Dies entspricht in etwa der KBV-Angabe für Q4/2017 mit $78 \%$ auf Basis der Mengendiskrepanz von 185.000 Tests zwischen der GOP 01737 mit 844.961 und der GOP 01738 mit 660.530 Leistungen

[15]. Aktuelle Praxis ist, dass die Labore die Entnahme- und Transportmaterialien des iFOBT kostenfrei für die Patienten der einsendenden Arztpraxen zur Verfügung stellen müssen.

- Relativ häufig wird durch die einsendende Praxis trotz entsprechender Indikation versäumt, „präventiv“ auf dem Anforderungsschein zu vermerken. Beispielsweise waren in den Laborzentren des LADR-Laborverbunds im Berichtszeitraum 15\% der iFOBT-Anforderungen kurativ.

- Weiterhin sind trotz entsprechender Aufklärungsunterlagen $4 \%$ der im Labor zurückerhaltenen Tests nicht durchführbar. Ursache war im Wesentlichen eine falsche Befüllung der Stuhlentnahmesysteme durch den Patienten. Hiervon waren anteilig an den nicht verwertbaren Stuhlproben die Entnahmesysteme einiger iFOBT häufiger betroffen: $63 \%$ Immundiagnostik, 19\% Mast/ Eiken, $16 \%$ Sysmex, 2 \% R-Biopharm. Abschließend sollte dies in folgenden Datenauswertungen weiter geklärt werden. Häufig kann die automatisierte Abarbeitung im medizinischen Labor auch durch eine nicht korrekte Anbringung des Patienten-Barcodes auf dem Entnahmesystem erschwert sein.

Zur Verbesserung der Früherkennungsmaßnahme muss damit sowohl die sachgerechte Verwendung als auch die Rückgabe des Tests durch den Patienten sowie 


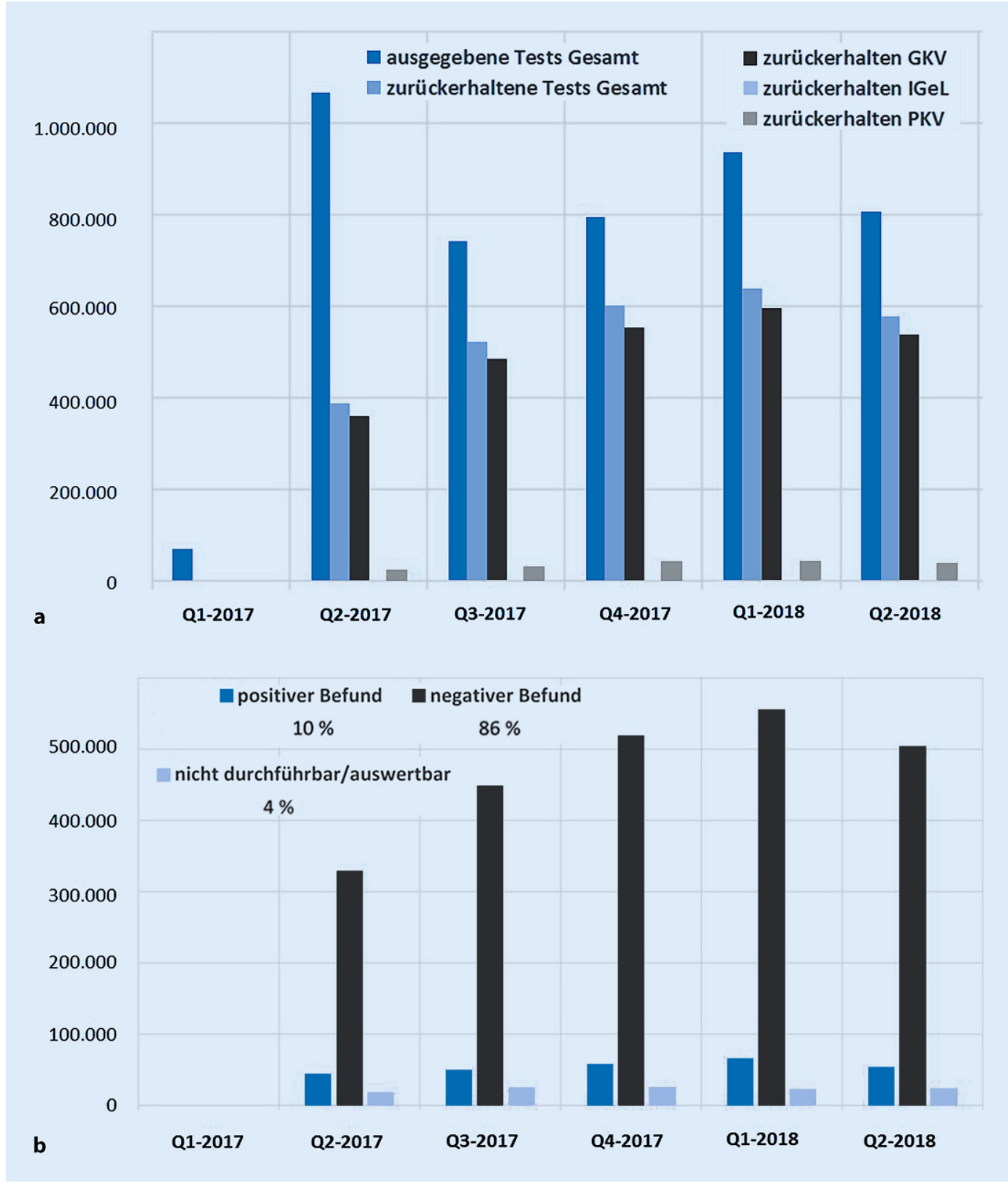

Abb. $1<$ a Insgesamt wurden durch die ALM(Akkreditierte Labore in der Medizin)-Mitgliedslabore 4.423.795 Mio. Stuhlentnahmesysteme für immunologische fäkale okkulte Bluttests (iFOBT) an Arztpraxen ausgegeben. Die Labore erhielten über den Untersuchungszeitraum 2.725.849 Stuhlproben (GKV [gesetzlich krankenversichert], PKV [privat krankenversichert] und IGeL [individuelle Gesundheitsleistung]) für den iFOBT zurück. Der Anteil an den zurückerhaltenden Proben betrug für den PKV-Bereich $<4 \%$ $(n=181.041)$ und für IGeL $<1 \%(n=8712)$. Nicht zurückerhalten wurden insgesamt in den Laboren 1.697.946 Entnahmesysteme für den iFOBT. Die kumulative Rücklaufquote über den untersuchten Zeitraum betrug damit $62 \%$. b Insgesamt zeigten $10 \%$ der analysierten iFOBT einen positiven ( $n=266.097$ ) und $86 \%$ einen negativen ( $n=2.349 .237)$ Befund. $4 \%$ der zurückerhaltenden Stuhlentnahmesysteme konnten nicht in einer Analyse verwertet werden $(n=110.514)$. (Unveröffentlichte Originaldaten, AG Versorgungsforschung des ALM e.V., Oktober 2018)

auch die Weiterleitung durch die Arztpraxis an das Labor verbessert werden.

Nur ein Teil der ALM-Mitgliedslabore hat im Hinblick auf die einsendenden Fachgebiete Daten geliefert. Die Verteilung der iFOBT-Einsendungen im Labor nach Fachgruppen war wie folgt: $34 \%$ Hausärztlich tätige Internisten und Allgemeinmediziner, $27 \%$ Fachärzte für Gynäkologie, $10 \%$ Fachärzte für Innere Medizin, $4 \%$ Fachärzte für Urologie und $<1 \%$ Fachärzte für Chirurgie. $25 \%$ der Anforderungen erfolgen durch sonstige, nicht für die präventive Testanforderung berechtigte Fachgruppen.
Die geschlechtsbezogene Auswertung der iFOBT-Anforderungen in den Laborzentren des LADR-Laborverbunds $(n=369.922)$ hat ergeben, dass die Verteilung der iFOBT-Anwendung bei Frauen und Männern 1,1:1 beträgt. Zu $2 \%$ wurden die präventiven iFOBT bei $<50$ Jahren angefordert, zu $26 \%$ bzw. $72 \%$ korrekt in den Altersgruppen 50-54 Jahre bzw. $>$ 54 Jahre.

\section{Schlussfolgerungen und vorgeschlagene Maßnahmen}

Der iFOBT wird im Vergleich zu den ehemals 4,7 Mio. Tests gFOBT pro Jahr [7] nicht ausreichend eingesetzt. Kumuliert man die Angabe der KBV von 660.530 durchgeführten Tests im QIV/2017 [15], so ergibt dies lediglich 2.642.120 iFOBT pro Jahr. Kumulativ erhielten die an diesem Bericht teilnehmenden ALM-Mitgliedslabore über das ausgewertete Jahr 2.536.096 iFOBT zurück. Weiterführende Untersuchungen müssen bestätigen, ob Frauen und Männer gleichmäßig in 
die Früherkennungsmaßnahme einbezogen werden. Das Anforderungsverhalten durch Fachärzte für Urologie ist beispielsweise ausbaufähig. Da ein hoher Anteil der Anforderung auch durch bisher nicht berechtigte Fachgruppen erfolgt, ist eine Aufhebung einer fachgebietsbezogenen Begrenzung zu diskutieren.

In Bezug auf die optimale Nutzung der Krebsfrüherkennung muss es die Aufgabe der Versorgungsforschung bleiben, zu evaluieren, welche Form der Patienteninformation zum Erfolg führt. Die zum 1. Juli 2019 geplanten Anschreiben im Rahmen eines organisierten Krebsfrüherkennungsprogramms sind ein richtiger Schritt [8]. Abzuwarten bleibt, ob der Beschluss, dass Abklärungskoloskopien nach einem positiven iFOBT als kurative (GOP 13421: 1766 Punkte, 188,15€) und nicht als Früherkennungskoloskopien (GOP 01741: 1945 Punkte, 207,23€) abzurechnen sind [16], den Erfolg des Screenings beeinflusst.

Die Zugänglichkeit einer Früherkennungsmaßnahme sollte flächendeckend sein. Ungünstig ist es, wenn regionale Verträge der Krankenkassen nur eine bundeslandbezogene Durchführung des iFOBT ermöglichen, die Labore aber Landes-KVen übergreifend in der Patientenversorgung tätig sind. Beispielsweise hat die AOK Nordost seit Oktober 2017 einen Vertrag zur Darmkrebsvorsorge für Patienten ab dem 40. Lebensjahr geschlossen [29], der nur eine Abrechnung gegenüber der KV-MecklenburgVorpommern für dort ansässige Labore ermöglicht. Labore aus anderen angrenzenden Bundesländern können mit ihrer Landes-KV den iFOBT nach der zugehörigen GOP 01738V bisher nicht abrechnen. Solche formalen Grenzen sollten für die Krebsfrüherkennung im Interesse der Patientenversorgung nicht bestehen.

Für die methodische Fortentwicklung des iFOBT ist es entscheidend, die Qualitätsindikatoren aus den Laborquartalsberichten an die KVen transparent testbezogen und im Hinblick auf die Labore pseudoanonym von zentraler Stelle zeitnah nach Übermittlung zu veröffentlichen. Insbesondere gilt dies auch für die sog. frühen Performanceindikatoren. Dabei sollte u.a. herausgearbeitet werden, welche Entnahmesysteme nicht so gut für den Patientengebrauch geeignet sind, um entsprechende Verbesserungen zu erwirken. Ebenfalls an zentraler Stelle sollten ggf. vorgenommene Anpassungen von Schwellenwerten durch Hersteller oder Anwender publiziert werden.

Weiterhin wäre eine Dokumentation der Qualität der Präanalytik sinnvoll, um einen Einfluss der Weiterleitungs- und Transportzeiten auf die Analyseergebnisse verfolgen zu können. Insgesamt gewährleisten die Labore eine Abholung der Proben auch im ländlichen Gebiet mit taggleichem Laboreingang und umgehender Analytik. Bezüglich der Diskrepanz zwischen den von den Laboren ausgegebenen und zurückerhaltenen Entnahmesystemen muss es aktuell noch ungeklärt bleiben, ob ein Test nicht vom Patienten zurückgebracht oder von der Arztpraxis nicht ins Labor weitergeleitet wurde [15]. Verluste von Tests auf dem Transportweg ins Labor sind den Laboren nur in sehr begrenzten Einzelfällen bekannt. Hinsichtlich des Probentransports wird zudem bei Abholung durch akkreditierte Labore u. a. eine temperaturgeführte Logistik beachtet, sodass keine Beeinflussung der Ergebnisse nach Jahreszeiten festgestellt werden konnte.

Um die Rücklaufquoten der Tests zur Laboranalytik nach Ausgabe an den Patienten für die Zukunft zu optimieren, kann diskutiert werden, ob eine direkte Kostenbeteiligung des Patienten am Entnahmesystem sinnvoll sein könnte. Im Rahmen der aktuellen Regelung der ausschließlichen Kostenübernahme für die Entnahmesysteme und Transportkosten durch die Labore entstanden allein mindestens 3 Mio. $€$ Kosten ohne Deckungsbeitrag über den 1-jährigen Berichtszeitraum durch nicht eingesendete Entnahmesysteme für die ALM-Mitgliedslabore, die Daten zu diesem Bericht geliefert haben. Offen bleibt somit grundsätzlich, ob die Bewertung des Entnahmesystems im Rahmen der GOP 01738 bzw. 32457 ausreichend vorgenommen wurde oder hierzu eine Anpassung vorgenommen werden muss.

\section{Fazit für die Praxis}

\section{- Die Durchführung der iFOBT ist gemäß den Qualitätsvorgaben in}

den akkreditierten medizinischen Laboren für die Routine in der Darmkrebsfrüherkennung etabliert.

- Die Auswertungen der von den Laboren an die KVen übermittelten Quartalsberichte sollten zentral veröffentlicht werden, um Optimierungen des Testeinsatzes gemeinsam mit den Anwendern und Herstellern voranzubringen.

- Die Aufklärung der Patienten erfolgt durch die den iFOBT ausgebende Ärzte in den Praxen. Entsprechende Vorgaben an die Patienten zur Handhabung des Entnahmesystems und zeitnahen Rückgabe sowie auch an das Praxispersonal zur korrekten Anforderung und Weiterleitung an das Labor sind entscheidend für die Qualität der Präanalytik.

- Die Beteiligung medizinischer Labore zur Fortentwicklung der Screeningprogramme und Bewertung der inhaltlichen Leistungen ist wünschenswert.

Korrespondenzadresse
Prof. Dr. med. Jan Kramer
LADR Der Laborverbund Dr.
Kramer \& Kollegen
Lauenburger Str. 67,
21502 Geesthacht,
Deutschland
j.kramer@LADR.de

\section{Einhaltung ethischer Richtlinien}

Interessenkonflikt. J. Kramer und U. Früh geben an, dass kein Interessenkonflikt besteht.

Dieser Beitrag beinhaltet keine von den Autoren durchgeführten Studien an Menschen oder Tieren.

Open Access. Dieser Artikel wird unter der Creative Commons Namensnennung 4.0 International Lizenz (http://creativecommons.org/licenses/by/4.0/deed. de) veröffentlicht, welche die Nutzung, Vervielfältigung, Bearbeitung, Verbreitung und Wiedergabe in jeglichem Medium und Format erlaubt, sofern Sie den/die ursprünglichen Autor(en) und die Quelle ordnungsgemäßnennen, einen Linkzur Creative Commons Lizenz beifügen und angeben, ob Änderungen vorgenommen wurden. 


\section{Literatur}

1. Ahlquist DA, McGill DB, Fleming JL, Schwartz $S$, Wieand HS, Rubin J, Moertel CG (1989) Patterns of occult bleeding in asymptomatic colorectal cancer. Cancer 63:1826-1830

2. AWMF (2013) S3 Leitlinie Kolorektales Karzinom, Version 1.0. AWMF-Registernummer:021/0070L

3. Brenner H, Tao S (2013) Superior diagnostic performance of faecal immunochemical tests for haemoglobin in a head-to-head comparison with guaiac based faecal occult blood test among 2235 participants of screening colonoscopy. Eur J Cancer 49:3049-3054

4. European Colorectal Cancer Screening Guidelines Working Group; von Karsa L, Patnick J, Segnan $\mathrm{N}$ et al (2013) European guidelines for quality assurance in colorectal cancer screening and diagnosis. Endoscopy 45(1):51-59. https://doi. org/10.1055/s-0032-1325997

5. Ferlay J, Soerjomataram I, Dikshit R, Eser S, Mathers C, Rebelo M, Parkin DM, Forman D, Bray F (2012) Cancer incidence and mortality worldwide: sources, methods and major patterns in GLOBOCAN. Int J Cancer 136:E359-E386

6. GBA, Gemeinsamer Bundesausschuss, Abteilung Methodenbewertung und veranlasste Leistungen (2016) Beschluss über eine Änderung der Krebsfrüherkennungs-Richtlinie: Bewertung eines iFOBT-basierten Darmkrebsscreenings im Vergleich zu einem gFOBT-basierten Darmkrebsscreening vom 21.04.2016. BAnz AT 08.07.2016 B2

7. GBA, Gemeinsamer Bundesausschuss, Abteilung Methodenbewertung und veranlasste Leistungen (2016) Zusammenfassende Dokumentation Beratungsverfahren gemäß $\S 25 \mathrm{a} S G B V$ in Verbindung mit $\S 135$ Abs. 1 SGB V, Bewertung eines iFOBTbasierten Darmkrebsscreenings im Vergleich zu einem gFOBT-basierten Darmkrebsscreening. BAnz 07.04.2016

8. GBA, Gemeinsamer Bundesausschuss, Abteilung Methodenbewertung und veranlasste Leistungen (2018) Beschluss des Gemeinsamen Bundesausschusses über eine Richtlinie für organisierte Krebsfrüherkennungsprogramme und eine Änderung der Krebsfrüherkennungs-Richtlinie vom 19. Juli2018. BAnz

9. Gies A, Cuk K, Schrotz-King P, Brenner H (2018) Direct comparison of diagnostic performance of 9 quantitative fecal Immunochemical tests for colorectal cancer screening. Gastroenterology 154:93-104

10. Greegor DH (1971) Occult blood testing for detection of asymptomatic colon cancer. Cancer 28:131-134

11. Guittet L, Bouvier V, Mariotte N, Vallee JP, Arsene D, Boutreux S, Tichet J, Launoy G (2007) Comparison of a guaiac based and an immunochemical faecal occult blood test in screening for colorectal cancer in a general average risk population. Gut 56:210-214

12. Hewitson $P$, Glasziou $P$, Watson $E$, Towler $B$, Irwig $L$ (2008) Cochrane systematic review of colorectal cancer screening using the fecal occult blood test (hemoccult): an update. Am J Gastroenterol 103:1541-1549

13. Hol $L$, van Leerdam $M E$, van Ballegooijen $M$, van Vuuren AJ, van Dekken H, Reijerink JC, van der Togt AC, Habbema JD, Kuipers EJ (2010) Screening for colorectal cancer: randomised trial comparing guaiac-based and immunochemical faecal occult blood testing and flexible sigmoidoscopy. Gut
59(1):62-68. https://doi.org/10.1136/gut.2009. 177089

14. Hundt S, Haug U, Brenner H (2009) Comparative evaluation of immunochemical fecal occult blood tests for colorectal adenoma detection. Ann Intern Med 150:162-169

15. KBV Analyse (2018) Viele immunologische Tests bleiben auf der Strecke. Ärzte Zeitung online 18.09.2018

16. KBV, Kassenärztliche Bundesvereinigung (2018) Koloskopie nach iFOBT gehöhrt nicht zur Früherkennung. Praxisnachrichten 15.03.2018

17. Krebsfrüherkennungsrichtlinie (KFE-RL) (2017) Richtlinie des Gemeinsamen Bundesausschusses über die Früherkennung von Krebserkrankungen vom 18.06.2009. In: BAnz 2009, Nr. 148a; geändert am 20. Juli 2017. BAnz AT 07.11.2017 B3

18. Lee JK, Liles EG, Bent S, Levin TR, Corley DA (2014) Accuracy of fecal immunochemical tests for colorectal cancer: systematic review and metaanalysis. Ann Intern Med 160:171

19. Mandel JS, Bond JH, Church TR, Snover DC, Bradley GM, Schuman LM, Ederer F (1993) Reducing mortality from colorectal cancer by screening for fecal occult blood. Minnesota Colon Cancer Control Study. N Engl J Med 328:1365-1371

20. Park DI, Ryu S, Kim YH, Lee SH, Lee CK, Eun CS, Han DS (2010) Comparison of guaiac-based and quantitative immunochemical fecal occult blood testing in a population at average risk undergoing colorectal cancer screening. Am J Gastroenterol 105:2017-2025

21. van Rossum LG, van Rijn AF, Laheij RJ, van Oijen $M G$, Fockens P, van Krieken HH, Verbeek AL, Jansen $J B$, Dekker E (2008) Random comparison of guaiac and immunochemical fecal occult blood tests for colorectal cancer in a screening population. Gastroenterology 135:82-90

22. van Rossum $L G$, van Rijn $A F$, Verbeek $A L$, van Oijen MG, Laheij RJ, Fockens P, Jansen JB, Adang EM, Dekker E (2011) Colorectal cancer screening comparing no screening, immunochemical and guaiac fecal occult blood tests: a cost-effectiveness analysis. Int J Cancer 128(8):1908-1917. https:// doi.org/10.1002/ijc. 25530

23. Scholefield JH, Moss SM, Mangham CM, Whynes DK, Hardcastle JD (2012) Nottingham trial of faecal occult blood testing for colorectal cancer: a 20-year follow-up. Gut 61(4):1036-1040. https://doi.org/ 10.1136/gutjnl-2011-300774

24. Schreuders EH, Ruco A, Rabeneck L, Schoen RE, Sung JJ, Young GP, Kuipers EJ (2015) Colorectal cancer screening: a global overview of existing programmes. Gut 64:1637-1649

25. Shaukat A, Mongin SJ, Geisser MS, Lederle FA, Bond JH, Mandel JS, Church TR (2013) Long-term mortality after screening for colorectal cancer. NEngl J Med 369:1106-1114

26. Starker A, Saß AC (2013) Inanspruchnahme von Krebsfrüherkennungsuntersuchungen: Ergebnisse der Studie zur Gesundheit Erwachsener in Deutschland (DEGS1). Bundesgesundheitsblatt Gesundheitsforschung Gesundheitsschutz 56(5-6):858-867

27. Starker A, Bertz J, Sass AC (2012) Inanspruchnahme von Krebsfrühereknnungsuntersuchungen. In: Robert Koch-Institut (Hrsg) Daten und Fakten: Ergebnisse der Studie "Gesundheit in Deutschland aktuell 2010“ Beiträge zur Gesundheitsbreichterstattung des Bundes. RKI, Berlin

28. Webendorfer S, Messerer P, Eberle F, Zober A (2004) Precautions for intestinal cancer in the workplace. An initiative for secondary prevention in the BASF joint-stock company. Dtsch Med Wochenschr 129:239-243

29. Wegner, J (2018) Vertrag zur Förderung der Darmkrebsfrüherkennung (Januar 2018). Journal Kassenärztliche Vereinigung MecklenburgVorpommern 27:7

30. Wieten E, Schreuders EH, Grobbee EJ, Nieboer D, Bramer WM, Lansdorp-Vogelaar I, Bruno MJ, Kuipers EJ, Spaander MCW (2018) Incidence of faecal occult blood test interval cancers in population-based colorectal cancer screening: a systematic review and meta-analysis. Gut 22(pii):gutjnl-2017-315340. https://doi.org/10. 1136/gutjnl-2017-315340

31. Zentralinstitut für die kassenärztliche Versorgung in der Bundesrepublik Deutschland (2016) Teilnahme an gesetzlichen Krebsfrüherkennungs untersuchungen bzw. Teilnahme an gesetzlichen Früherkennungsuntersuchungen (fäkaler okkulter Bluttest (FOBT), Koloskopie) und an Beratungen zur Prävention von Darmkrebs. http://www.gbebund.de (Erstellt: 14. Okt. 2018). Zugegriffen: 14. Okt. 2018

32. Zentrum für Krebsregisterdaten im Robert KochInstitut (Hrsg) (2016) RKI-Bericht zum Krebsgeschehen in Deutschland. RKI, Berlin

33. Zhu MM, Xu XT, Nie F, Tong JL, Xiao SD, Ran ZH (2010) Comparison of immunochemical and guaiac-based fecal occult blood test in screening and surveillance for advanced colorectal neoplasms: a meta-analysis. J Dig Dis 11:148-160 